Network Working Group

Request for Comments: 4318

Category: Standards Track
D. Levi

Nortel Networks

D. Harrington Effective Software

December 2005

\title{
Definitions of Managed Objects for Bridges
}

with Rapid Spanning Tree Protocol

\section{Status of This Memo}

This document specifies an Internet standards track protocol for the Internet community, and requests discussion and suggestions for improvements. Please refer to the current edition of the "Internet Official Protocol Standards" (STD 1) for the standardization state and status of this protocol. Distribution of this memo is unlimited.

Copyright Notice

Copyright (C) The Internet Society (2005).

Abstract

This memo defines an SMIv2 MIB module for managing the Rapid spanning Tree capability defined by the IEEE P802.1t and P802.1w amendments to IEEE Std 802.1D-1998 for bridging between Local Area Network (LAN) segments. The objects in this MIB are defined to apply both to transparent bridging and to bridges connected by subnetworks other than LAN segments.

Table of Contents

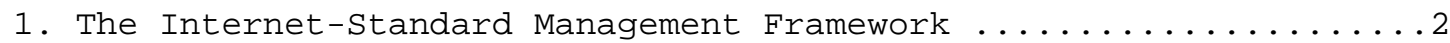

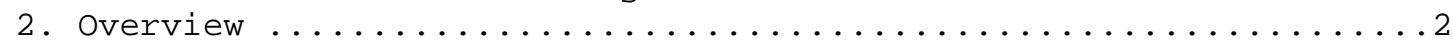

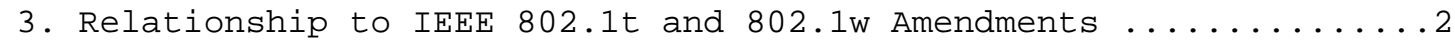

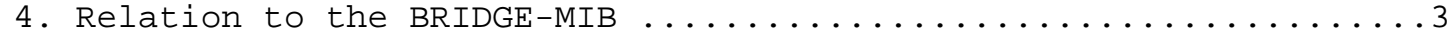

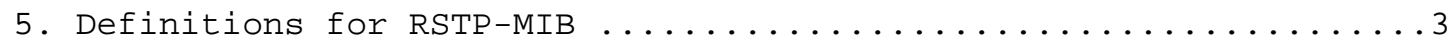

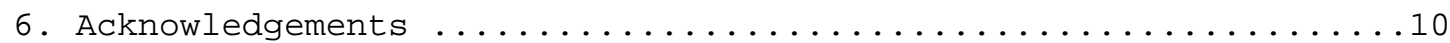

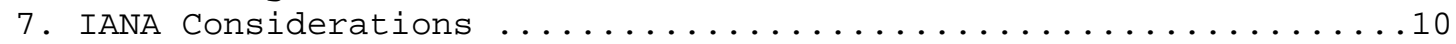

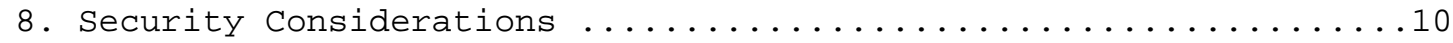

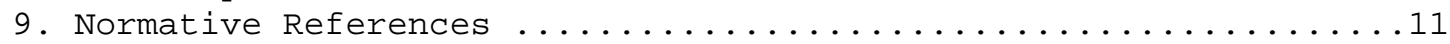

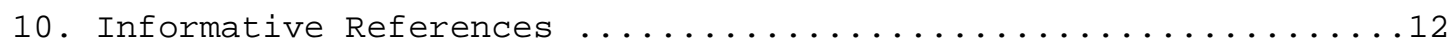


1. The Internet-Standard Management Framework

For a detailed overview of the documents that describe the current Internet-Standard Management Framework, please refer to section 7 of RFC 3410 [RFC3410].

Managed objects are accessed via a virtual information store, termed the Management Information Base or MIB. MIB objects are generally accessed through the Simple Network Management Protocol (SNMP). Objects in the MIB are defined using the mechanisms defined in the Structure of Management Information (SMI). This memo specifies a MIB module that is compliant to the SMIV2, which is described in STD 58, RFC 2578 [RFC2578], STD 58, RFC 2579 [RFC2579] and STD 58, RFC 2580 $[\mathrm{RFC} 2580]$.

\section{Overview}

This memo defines an SMIv2 MIB module for managing the Rapid spanning Tree (RSTP) capability defined by the IEEE P802.1t [802.1t] and P802.1w [802.1w] amendments to IEEE Std 802.1D-1998 [802.1D-1998] for bridging between Local Area Network (LAN) segments. The objects in this MIB are defined to apply both to transparent bridging and to bridges connected by subnetworks other than LAN segments.

3. Relationship to IEEE 802.1t and 802.1w Amendments

This document defines managed objects for the Rapid Spanning Tree Protocol defined by the IEEE P802.1t and IEEE P802.1w amendments to $802.1 \mathrm{D}-1998$.

RSTP-MIB Name

$\operatorname{dot} 1 \mathrm{dStp}$

dot1dStpVersion

dot 1 dStpTxHoldCount

dotldStpextPortTable dot1dStpPortProtocolMigration dot1dStpPortAdminEdgePort dot1dStpPortoperEdgePort dot1dStpPortAdminPointToPoint dot1dStpPortoperPointToPoint dot1dStpPortAdminPathCost
IEEE 802.1 Reference

(w) 17.16 .1 ForceVersion

(w) 17.16 .6 TxHoldCount

(w) 17.18 .10 mcheck

(t) 18.3 .3 adminEdgePort

(t) 18.3 .4 operEdgePort

(w) 6.4 .3 adminPointToPointMAC

(w) 6.4 .3 operPointToPointMAC

(D) 8.5 .5 .3 Path Cost

There are concerns that there may be changes made in the 802.1D-2004 edition that would lead to non-backward-compatible SMI changes for 802.1t and 802.1w managed objects in the MIB modules. The Bridge MIB working group decided to 'freeze' the technical content of the MIB modules at a level that is compatible with the $802.1 \mathrm{t}$ and $802.1 \mathrm{w}$ 


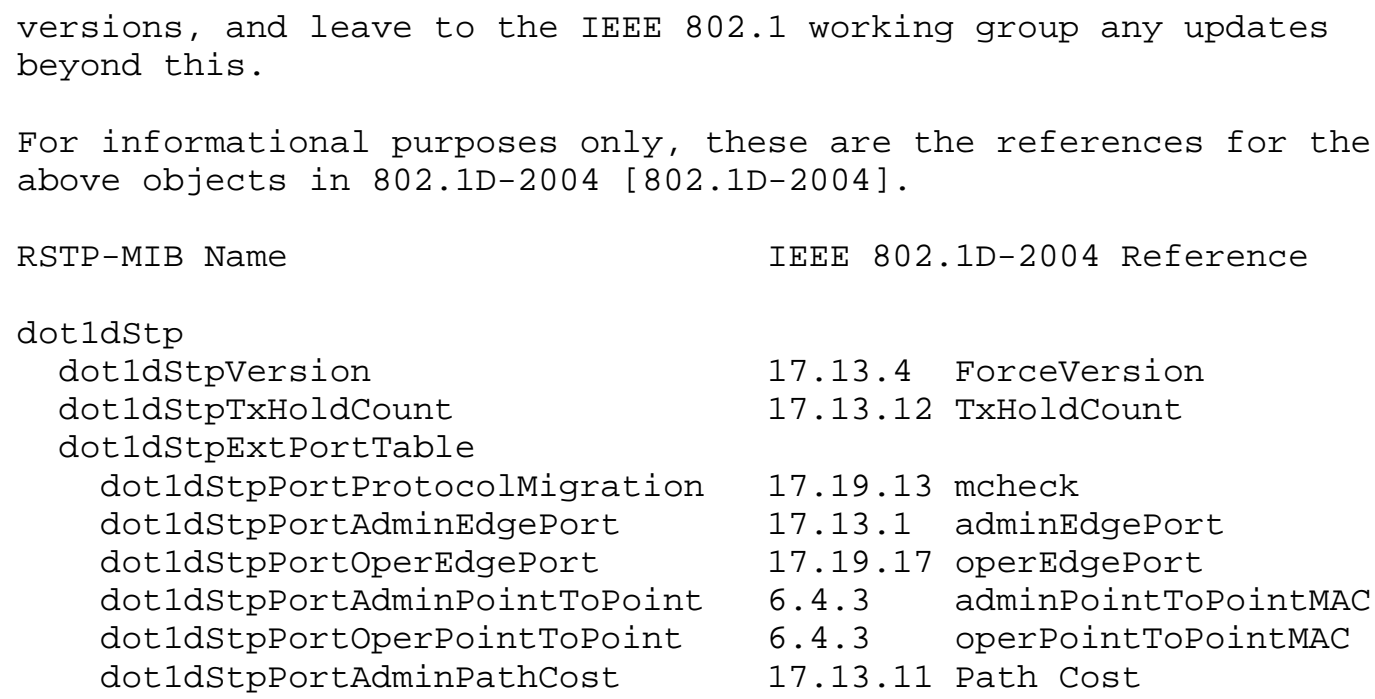

4. Relation to the BRIDGE-MIB

The objects in the RSTP-MIB supplement those defined in the Bridge MIB [RFC4188].

The Original BRIDGE-MIB [RFC1493] has been updated in an SMIv2compliant version [RFC4188]. Conformance statements have been added and some description and reference clauses have been updated. The interpretations of some objects were changed to accommodate IEEE $802.1 \mathrm{t}$ and $802.1 \mathrm{w}$ amendments.

The object dot1dStpPortPathCost32 was added to support IEEE 802.1t, and the permissible values of dotldstppriority and dot1dStpPortPriority have been clarified for bridges supporting IEEE 802.1t or IEEE 802.1w. The interpretation of dot1dStpTimesinceTopologychange has been clarified for bridges supporting the RSTP.

See the updated BRIDGE-MIB [RFC4188] for details.

5. Definitions for RSTP-MIB

RSTP-MIB DEFINITIONS : := BEGIN

-- MIB for IEEE 802.1w Rapid Spanning Tree Protocol

IMPORTS 


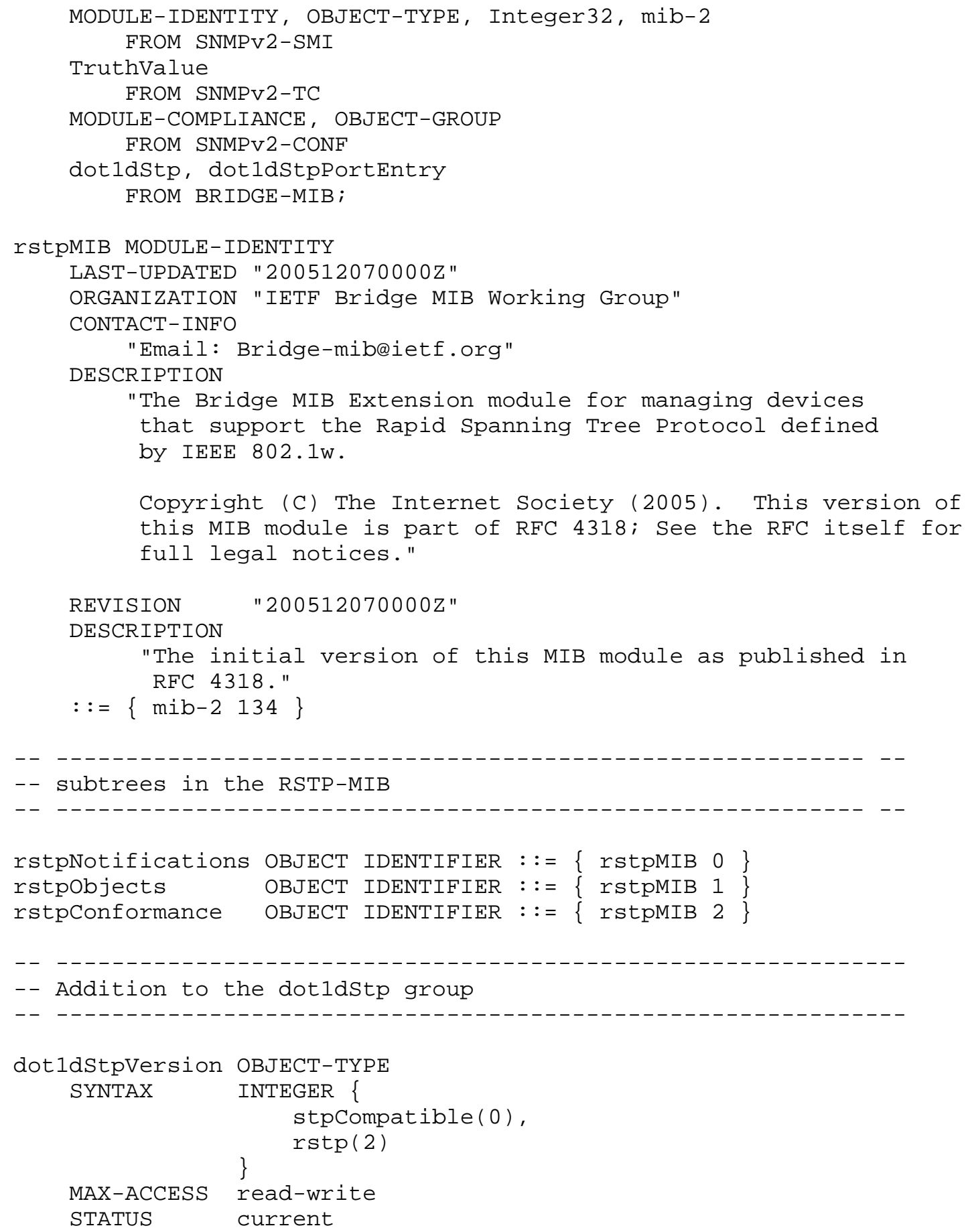




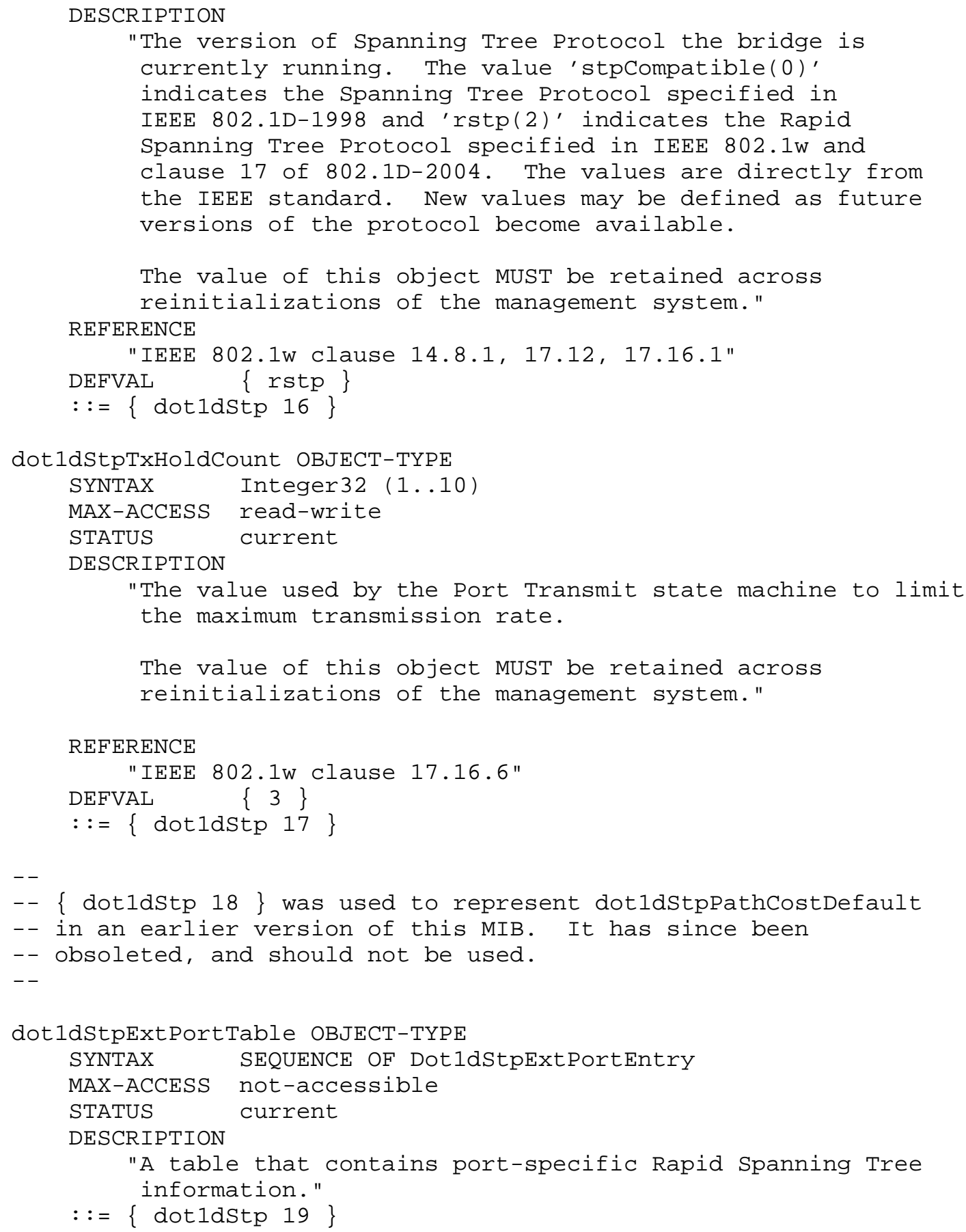




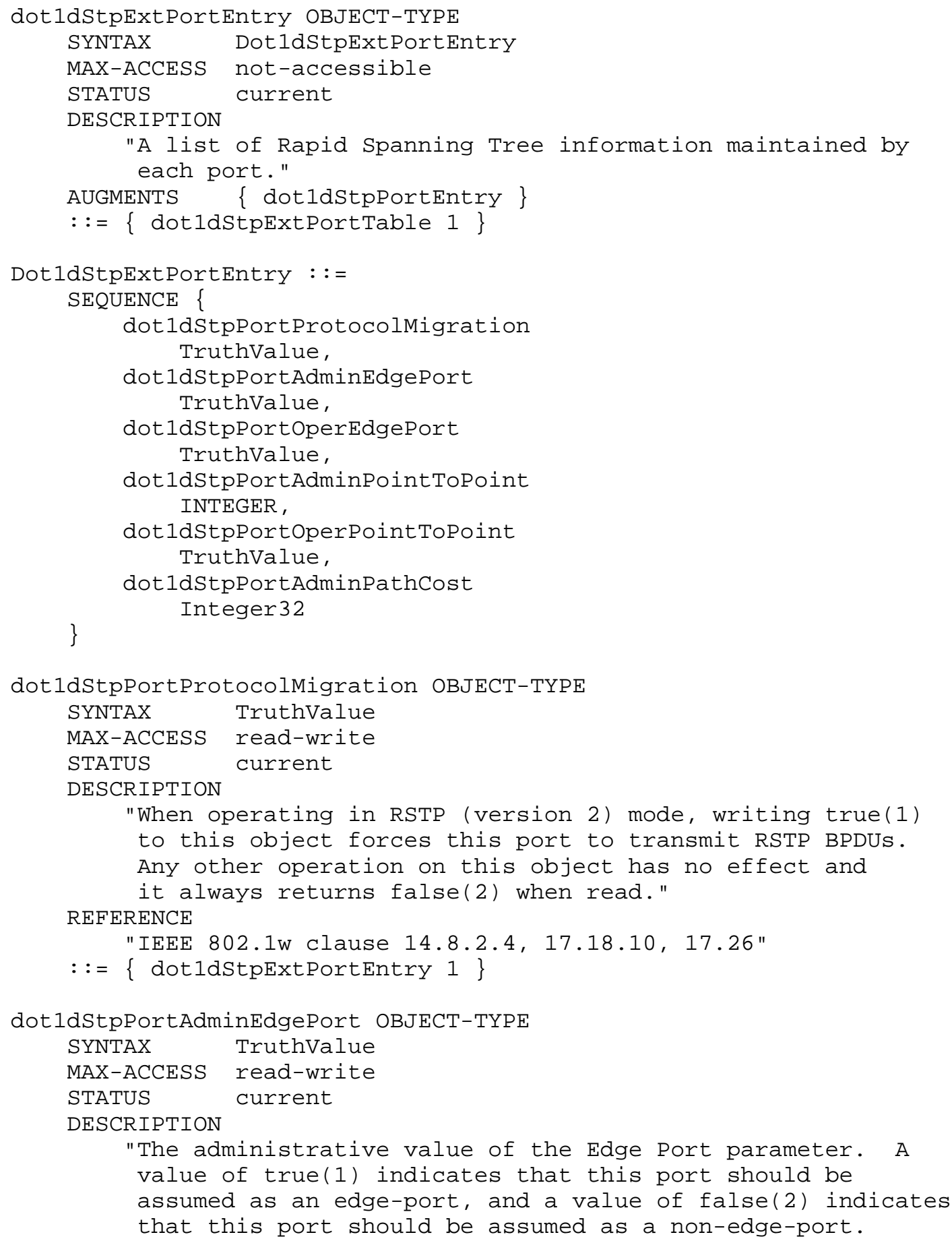




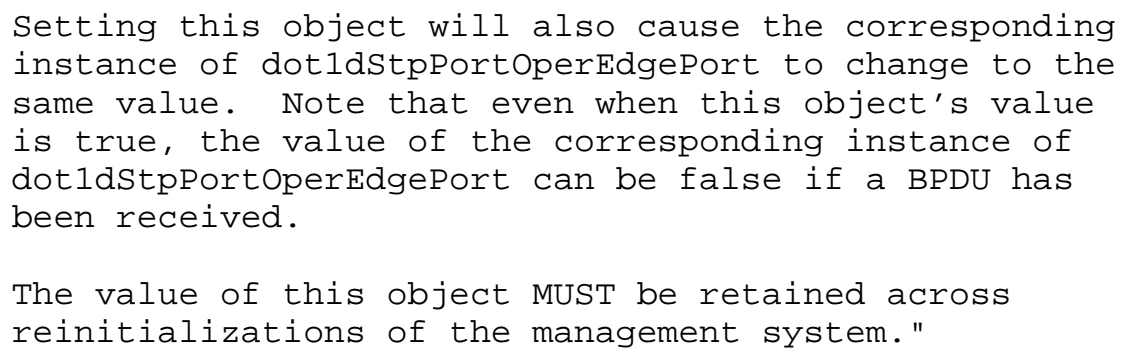




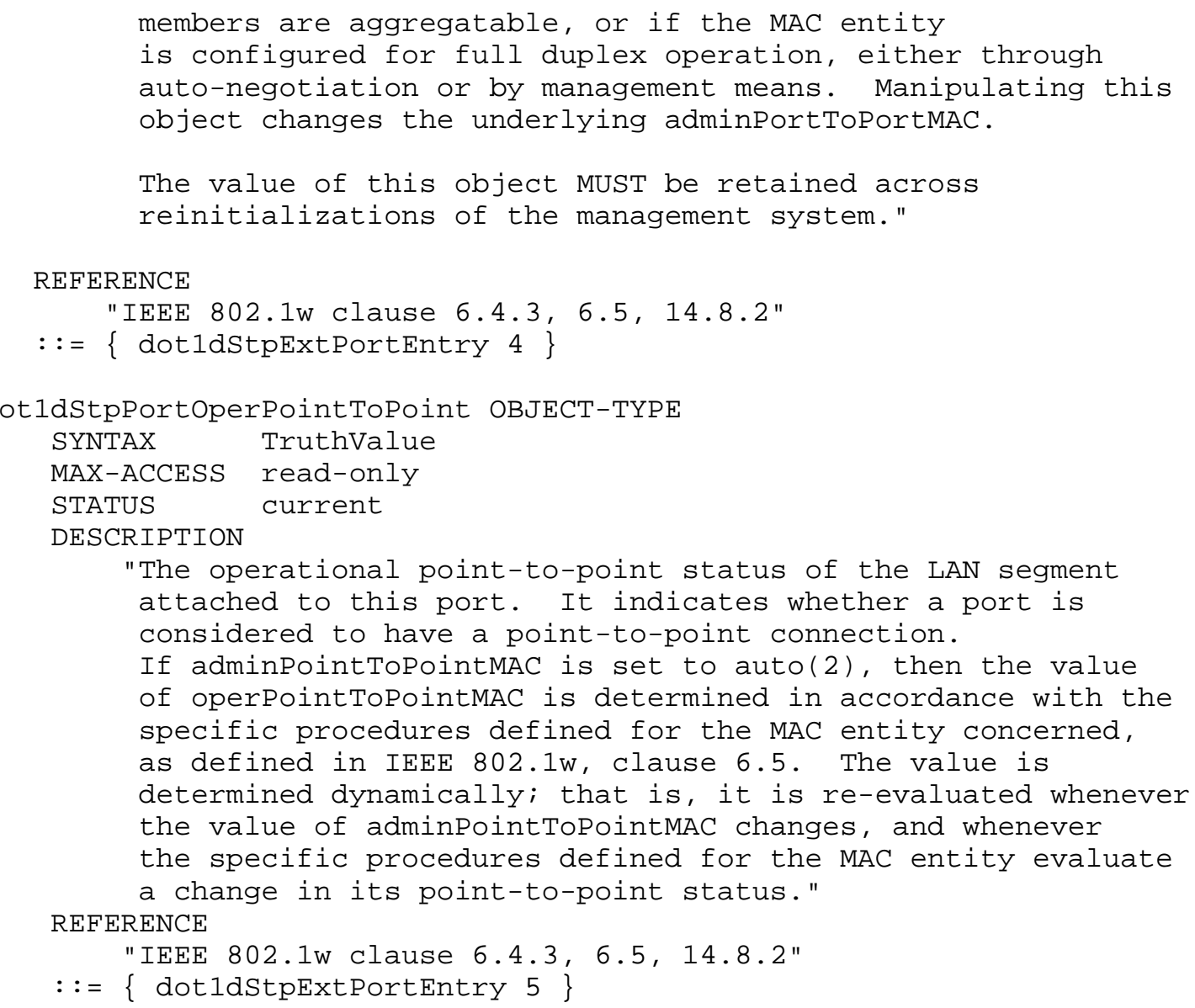

dot 1dStpPortAdminPathCost OBJECT-TYPE

SYNTAX Integer32 (0..200000000)

MAX-ACCESS read-write

STATUS current

DESCRIPTION

"The administratively assigned value for the contribution of this port to the path cost of paths toward the spanning tree root.

Writing a value of ' 0 ' assigns the automatically calculated default Path Cost value to the port. If the default Path Cost is being used, this object returns ' 0 ' when read.

This complements the object dot1dStpPortPathCost or dot1dStpPortPathCost32, which returns the operational value of the path cost. 


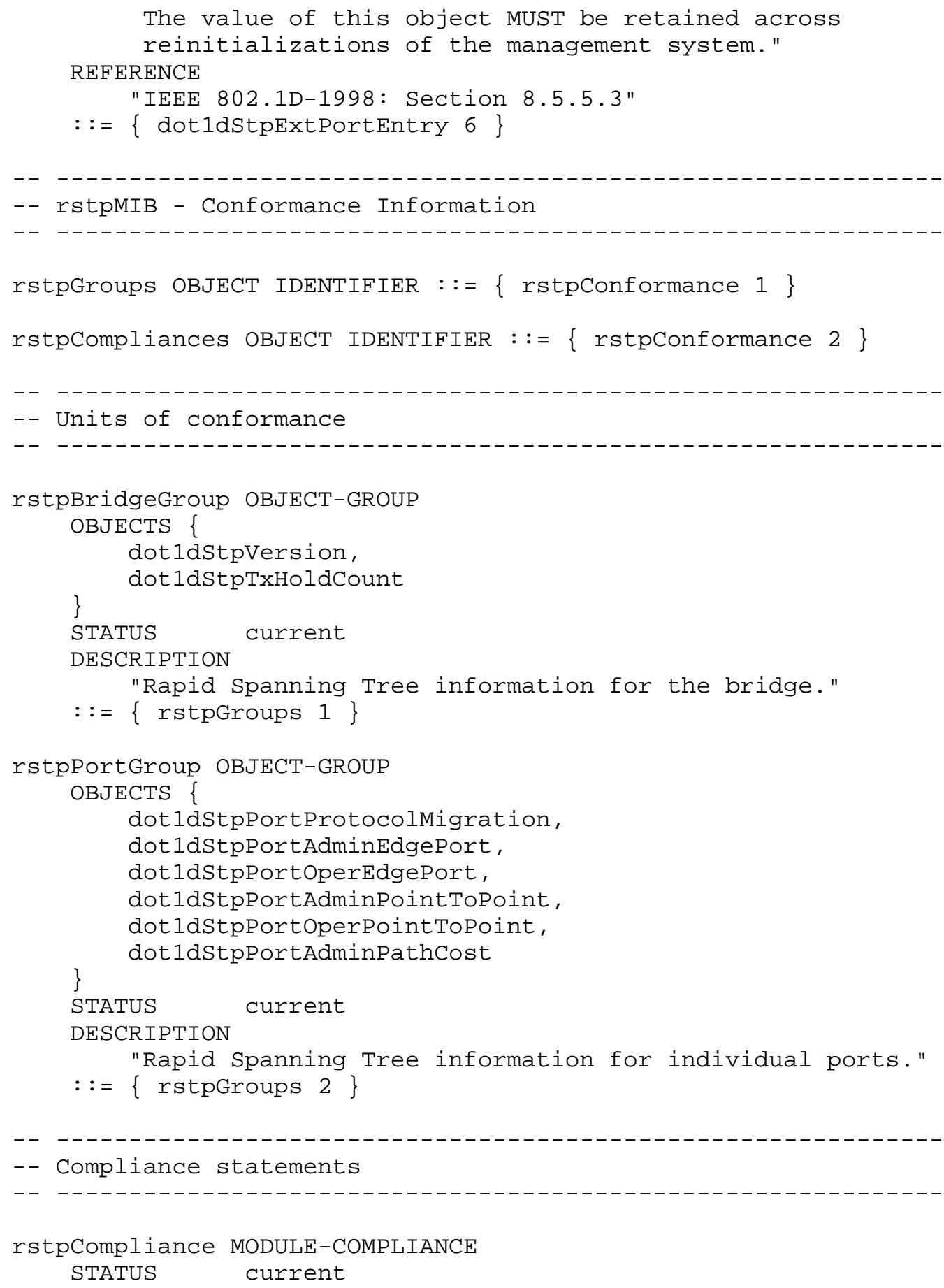




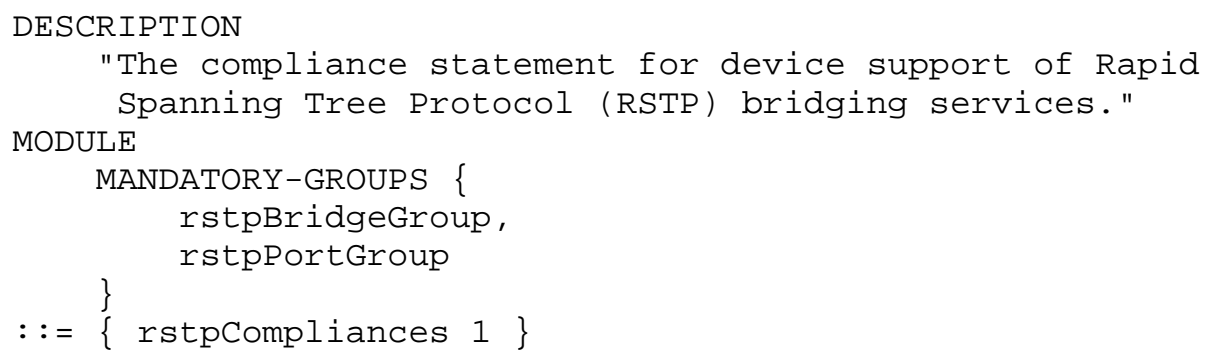

END

\section{Acknowledgements}

This document was produced on behalf of the Bridge MIB Working Group in the Operations and Management area of the Internet Engineering Task Force.

The authors wish to thank the members of the Bridge MIB Working Group, especially Alex Ruzin, for their comments and suggestions that improved this effort.

Vivian Ngai and Les Bell were the initial authors of this document, and did the bulk of the development work for this document.

7. IANA Considerations

The IANA has assigned the following OID:

$$
\begin{array}{ll}
\text { Descriptor } & \text { OBJECT IDENTIFIER value } \\
--------- & ------------------- \\
\text { rstpMIB } & \{\text { mib-2 } 134\}
\end{array}
$$

8. Security Considerations

There are a number of management objects defined in this MIB module with a MAX-ACCESS clause of read-write and/or read-create. Such objects may be considered sensitive or vulnerable in some network environments. The support for SET operations in a non-secure environment without proper protection can have a negative effect on network operations. These are the tables and objects and their sensitivity/vulnerability:

Writable objects that could be misused to cause network delays and spanning tree instabilities include dotldStpVersion, dot1dStpTxHoldCount, dot1dStpPortProtocolMigration, dot1dStpPortAdminEdgePort, and dot1dStpPortAdminPathCost. 
Some of the readable objects in this MIB module (i.e., objects with a MAX-ACCESS other than not-accessible) may be considered sensitive or vulnerable in some network environments. It is thus important to control even GET and/or NOTIFY access to these objects and possibly to even encrypt the values of these objects when sending them over the network via SNMP. These are the tables and objects and their sensitivity/vulnerability:

dotldStpVersion could be read by an attacker to identify environments containing applications or protocols that are potentially sensitive to RSTP mode.

dot1dStpPortAdminPointToPoint could be used to mislead an access control protocol, such as $802.1 x$, to believe that only one other system is attached to a LAN segment and to enable network access based on that assumption. This situation could permit potential man-in-the-middle attacks.

SNMP versions prior to SNMPV3 did not include adequate security. Even if the network itself is secure (for example by using IPsec), even then, there is no control as to who on the secure network is allowed to access and GET/SET (read/change/create/delete) the objects in this MIB module.

It is RECOMMENDED that implementers consider the security features as provided by the SNMPV3 framework (see [RFC3410], section 8), including full support for the SNMPv3 cryptographic mechanisms (for authentication and privacy).

Further, deployment of SNMP versions prior to SNMPv3 is NOT RECOMMENDED. Instead, it is RECOMMENDED to deploy SNMPv3 and to enable cryptographic security. It is then a customer/operator responsibility to ensure that the SNMP entity giving access to an instance of this MIB module is properly configured to give access to the objects only to those principals (users) that have legitimate rights to indeed GET or SET (change/create/delete) them.

9. Normative References

[RFC2578] MCCloghrie, K., Perkins, D., and J. Schoenwaelder, "Structure of Management Information Version 2 (SMIv2)", STD 58, RFC 2578, April 1999.

[RFC2579] MCCloghrie, K., Perkins, D., and J. Schoenwaelder, "Textual Conventions for SMIv2", STD 58, RFC 2579, April 1999. 
[RFC2580] McCloghrie, K., Perkins, D., and J. Schoenwaelder, "Conformance Statements for SMIv2", STD 58, RFC 2580, April 1999.

[802.1D-1998] "Information technology - Telecommunications and information exchange between systems - Local and metropolitan area networks - Common specifications Part 3: Media Access Control (MAC) Bridges: Revision. This is a revision of ISO/IEC 10038: 1993, 802.1j-1992 and $802.6 \mathrm{k}-1992$. It incorporates P802.11c, P802.1p and P802.12e." ISO/IEC 15802-3: 1998.

[RFC4188] Norseth, K. and E. Bell, "Definitions of Managed objects for Bridges", RFC 4188, September 2005.

[802.1t] IEEE 802.1t-2001, " (Amendment to IEEE Standard 802.1D) IEEE Standard for Information technology Telecommunications and information exchange between systems - Local and metropolitan area networks - Common specifications - Part 3: Media Access Control (MAC) Bridges: Technical and Editorial Corrections".

[802.1w] IEEE 802.1w-2001, " (Amendment to IEEE Standard 802.1D) IEEE Standard for Information technology-Telecommunications and information exchange between systems--Local and metropolitan area networks--Common Specifications--Part 3: Media Access Control (MAC) Bridges: Rapid Reconfiguation".

10. Informative References

[RFC3410] Case, J., Mundy, R., Partain, D., and B. Stewart, "Introduction and Applicability Statements for Internet-Standard Management Framework", RFC 3410, December 2002 .

[802.1D-2004] IEEE Project 802 Local and Metropolitan Area Networks, "IEEE Standard 802.1D-2004 MAC Bridges", 2004.

[RFC1493] Decker, E., Langille, P., Rijsinghani, A., and K. McCloghrie, "Definitions of Managed Objects for Bridges", RFC 1493, July 1993. 


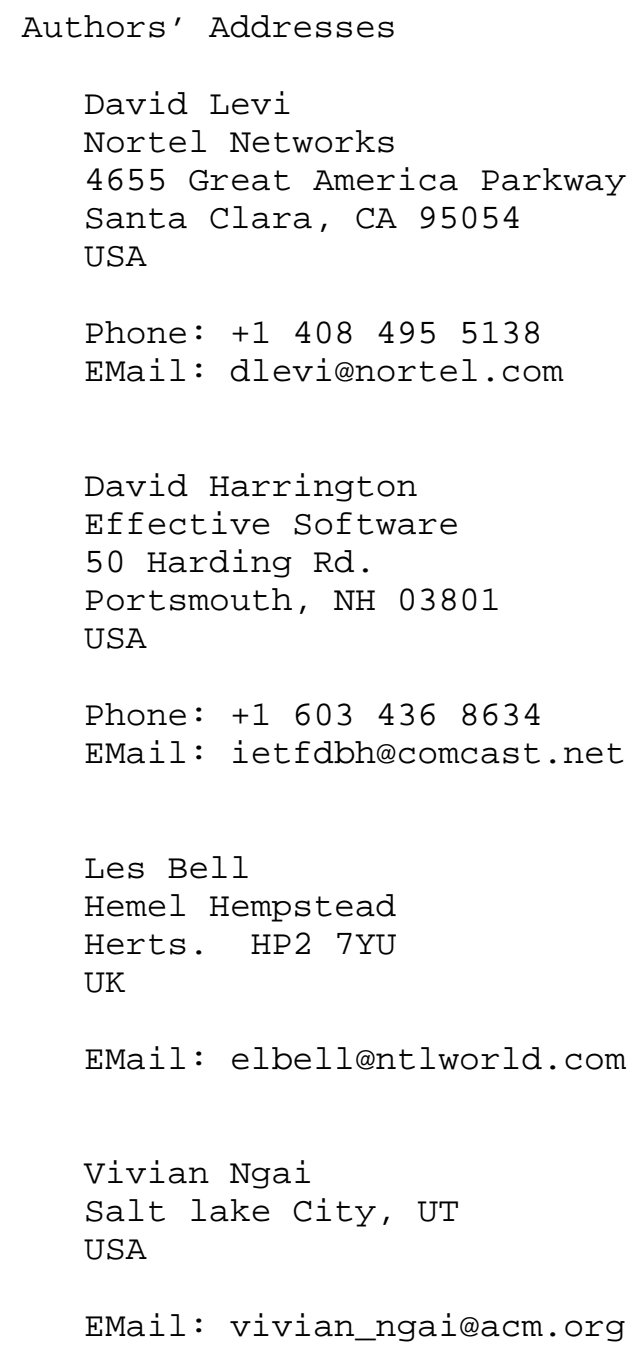


Full Copyright statement

Copyright (C) The Internet Society (2005).

This document is subject to the rights, licenses and restrictions contained in BCP 78, and except as set forth therein, the authors retain all their rights.

This document and the information contained herein are provided on an "AS IS" basis and THE CONTRIBUTOR, THE ORGANIZATION HE/SHE REPRESENTS OR IS SPONSORED BY (IF ANY), THE INTERNET SOCIETY AND THE INTERNET ENGINEERING TASK FORCE DISCLAIM ALL WARRANTIES, EXPRESS OR IMPLIED, INCLUDING BUT NOT LIMITED TO ANY WARRANTY THAT THE USE OF THE INFORMATION HEREIN WILL NOT INFRINGE ANY RIGHTS OR ANY IMPLIED WARRANTIES OF MERCHANTABILITY OR FITNESS FOR A PARTICULAR PURPOSE.

Intellectual Property

The IETF takes no position regarding the validity or scope of any Intellectual Property Rights or other rights that might be claimed to pertain to the implementation or use of the technology described in this document or the extent to which any license under such rights might or might not be available; nor does it represent that it has made any independent effort to identify any such rights. Information on the procedures with respect to rights in RFC documents can be found in BCP 78 and BCP 79 .

Copies of IPR disclosures made to the IETF Secretariat and any assurances of licenses to be made available, or the result of an attempt made to obtain a general license or permission for the use of such proprietary rights by implementers or users of this specification can be obtained from the IETF on-line IPR repository at http://www.ietf.org/ipr.

The IETF invites any interested party to bring to its attention any copyrights, patents or patent applications, or other proprietary rights that may cover technology that may be required to implement this standard. Please address the information to the IETF at ietfipreietf.org.

Acknowledgement

Funding for the RFC Editor function is currently provided by the Internet Society. 